\title{
Consumption of ultra-processed foods and health outcomes: a systematic review of epidemiological studies
}

Xiaojia Chen ${ }^{1,2+}$, Zhang Zhang ${ }^{1,2+}$, Huijie Yang ${ }^{1,2+}$, Peishan Qiu ${ }^{1,2}$, Haizhou Wang ${ }^{1,2}$, Fan Wang ${ }^{1,2}$, Qiu Zhao ${ }^{1,2^{*}}$ (D), Jun Fang ${ }^{1,2^{*}}$ and Jiayan $\mathrm{Nie}^{1,2^{*}}$

\begin{abstract}
Background: Consumption of ultra-processed foods (UPFs) plays a potential role in the development of obesity and other diet-related noncommunicable diseases (NCDs), but no studies have systematically focused on this. This study aimed to summarize the evidence for the association between UPFs consumption and health outcomes.

Methods: A comprehensive search was conducted in PubMed, Embase, and Web of Science to identify all relevant studies. Epidemiological studies were included, and identified studies were evaluated for risk of bias.A narrative review of the synthesized findings was provided to assess the association between UPFs consumption and health outcomes.

Results: 20 studies (12 cohort and 8 cross-sectional studies) were included in the analysis, with a total of 334,114 participants and 10 health outcomes. In a narrative review, high UPFs consumption was obviously associated with an increased risk of all-cause mortality, overall cardiovascular diseases, coronary heart diseases, cerebrovascular diseases, hypertension, metabolic syndrome, overweight and obesity, depression, irritable bowel syndrome, overall cancer, postmenopausal breast cancer, gestational obesity, adolescent asthma and wheezing, and frailty. It showed no significant association with cardiovascular disease mortality, prostate and colorectal cancers, gestational diabetes mellitus and gestational overweight.
\end{abstract}

Conclusions: This study indicated a positive association between UPFs consumption and risk of several health outcomes. Large-scale prospective designed studies are needed to confirm our findings.

Keywords: Ultra-processed foods, Noncommunicable diseases, Health, Systematic review

\section{Introduction}

Noncommunicable diseases (NCDs), such as cardiovascular diseases, type 2 diabetes and some cancers, are collectively responsible for almost $70 \%$ of all deaths worldwide. The current prevalence of NCDs poses

\footnotetext{
* Correspondence: zhaoqiuwhu@163.com; xhfangjun@163.com;

119140546@qq.com

${ }^{\top}$ Xiaojia Chen, Zhang Zhang and Huijie Yang contributed equally to this work.

'Department of Gastroenterology, Zhongnan Hospital of Wuhan University, No. 169, Donghu Road, Wuchang District, Wuhan 430071, Hubei Province, China

Full list of author information is available at the end of the article
}

devastating health outcomes and constitutes a serious threat to global health systems. To reduce the number of deaths caused by NCDs, a better understanding of the potential risk factors is needed.

Unhealthy diets are recognized as a major determinant of the occurrence of NCDs. With the increasing trend of NCDs, a steady rise in the share of processing foods has been seen. In the last half century food processing has evolved greatly as a consequence of the industrialization and globalization of food systems [1]. Negative effects on nutritional dietary quality emerged subsequently, such as higher content in free sugars, saturated fats, energy

(C) The Author(s). 2020 Open Access This article is licensed under a Creative Commons Attribution 4.0 International License, which permits use, sharing, adaptation, distribution and reproduction in any medium or format, as long as you give appropriate credit to the original author(s) and the source, provide a link to the Creative Commons licence, and indicate if changes were made. The images or other third party material in this article are included in the article's Creative Commons licence, unless indicated otherwise in a credit line to the material. If material is not included in the article's Creative Commons licence and your intended use is not permitted by statutory regulation or exceeds the permitted use, you will need to obtain permission directly from the copyright holder. To view a copy of this licence, visit http://creativecommons.org/licenses/by/4.0/ The Creative Commons Public Domain Dedication waiver (http://creativecommons.org/publicdomain/zero/1.0/) applies to the data made available in this article, unless otherwise stated in a credit line to the data. 
density and sodium, and less content in protein, fiber and micronutrients. It is believed that most NCDs can be prevented by changes in diet patterns.

Ultra-processed foods (UPFs) are defined as formulations of ingredients derived from foods and additives, coupled with substances including colorings, flavorings, sweeteners, and emulsifiers [2]. They contain little if any intact food. Included in this definition are sugarsweetened beverages, sweets, ice cream, chocolates, savoury snacks, burgers, processed meat and frozen dishes. Compared with other food groups, UPFs are typically durable, ready to consume, low-cost and hyperpalatable. They tend to be packaged delicately and marketed concentratedly. They are characteristically fatty, sugary or salty, energy-dense and lack of protein, dietary fibre, micronutrients and several bioactive compounds [3-5]. Furthermore, they may contain neo-formed contaminants derived from industrial processing, as well as substances from additives and packaging [6, 7]. Considering the association between UPFs and poorer dietary quality, the share of UPFs has been proposed as an effective predictor of population diet quality [8-10].

The whole world has witnessed a dramatic transition in food consumption patterns. Unprocessed or minimally processed foods and freshly prepared meals are gradually displaced by UPFs. The shift appeared initially in high and middle income countries, and then worldwide $[11,12]$. Transnational corporations are major factors that drive the production and sales of UPFs, along with their convenience, branding and aggressive marketing [13]. These characteristics create massive market advantages for UPFs over other food groups [14]. In high income countries, more than half of the foods consumed are UPFs for most of the age groups, and consumption decreases with age $[15,16]$. Purchase surveys and dietary trends on UPFs consumption have been performed in Asia and many western countries [17-21]. It has been evaluated that the energy contribution of UPFs ranged from 25 to $60 \%$ [22].

The existing evidence indicates that displacement by UPFs is driving a rising prevalence of obesity and other diet-related NCDs [23]. A growing body of evidence suggests that increases of UPFs in dietary proportion were associated with a higher incidence of adverse health outcomes [16, 24-42]. Decreasing the dietary share of UPFs may notably contribute to the prevention of diet-related NCDs [43-45].

As UPFs are increasing dominantly during the past decades, understanding their potential impacts on health outcomes has become a major imperative. To date, however, this literature has not been comprehensively evaluated. No reviews have been conducted on this topic previously. To address these concerns, this systematic review was conducted to summarize the evidence for the association between UPFs consumption and health outcomes.

\section{Methods}

\section{Study design}

This systematic review is completed according to the MOOSE (Meta-analysis Of Observational Studies in Epidemiology) Statement [46]. We developed a protocol with methods of the review in advance (Supplement 1).

\section{Search strategy}

The public databases of PubMed, Embase, and Web of Science were comprehensively searched for relevant studies published up to October 11, 2019. Broad search strategy was used to ensure that no publications were overlooked. The search terms were listed in Supplement 1. Studies in language other than English were excluded. Reference lists of relevant articles and some key journals were also hand-searched for other pertinent studies. We considered no limitations on the publication date.

\section{Eligibility criteria}

Epidemiological studies including cohort and crosssectional studies were considered for further screening. Eligibility was assessed independently by two authors (Xiaojia Chen and Zhang Zhang). All differences were resolved by consensus with a third author (Fan Wang). We included studies meeting the following inclusion criteria: (i) included more than 500 participants; (ii) the exposure of interest was consumption of UPFs and the outcomes of interest were any health outcomes (e.g., allcause mortality, cancers); (iii) reported the effect sizes of hazard ratios (HRs), odds ratios (ORs) or relative risks (RRs) with 95\% confidence intervals (CIs). We excluded experimental studies, reviews, letters, editorials, and abstracts without full texts. When more than one studies reported on the same cohort and outcome, we only included the study with the longest follow-up.

\section{Data extraction}

Both authors independently reviewed full-texts of the eligible studies and extracted data using a standardized collection form. All differences were resolved by consensus. Information extracted from each study was as follows: first author, year of publication, study design, study period and area, study population, number of participants, exposure assessment, outcome measures and categories, comparison, effect sizes (HRs, ORs, and RRs) with 95\% CIs. All kinds of measures of exposures and outcomes were allowed. There was also no limitation on outcome categories. 


\section{Quality assessment}

Risk of bias was evaluated by two reviewers independently. All differences were resolved by consensus. We assessed the quality of cohort studies with the Newcastle-Ottawa Scale (NOS) and cross-sectional studies with the JBI Critical Appraisal Checklist $[47,48]$. Risk of bias of each eligible study was evaluated according to a series of methodological features: (i) sampling of participants and their representativeness of the population; (ii) assessment of exposure to UPFs; (iii) ascertainment of health outcomes; (iv) adjustment for potential confounders; (v) demonstration was mentioned that outcome of interest was not present at start of study. In general, cohort studies scoring $\geq 6$ were considered as high quality, while cross-sectional studies with $\geq 5$ "yes" were rated as high quality.

\section{Data synthesis}

In light of the overall low number of studies, variance of exposures and outcomes measurement, no quantitative meta-analysis was conducted. To systematically synthesize findings across included studies, a narrative synthesis approach was chosen. We tabulated study characteristics and classified studies into groups according to different health outcomes. The evidence was synthesized to provide useful insights for the association of interest.

\section{Results}

\section{Study selection and characteristics}

The search strategy identified 1165 records. 563 articles were screened by titles and abstracts after duplicates removed. Of the 55 full-texts assessed for eligibility, 20 published epidemiological studies (12 cohort and 8 cross-sectional studies) were included into the systematic review, with a total of 334,114 participants and 10 diseases (Fig. 1). An overview of the characteristics of included studies was provided in Table 1 . All studies were published between 2015 and 2019, with a sample size ranging from 785 to 109,104 . Six studies were conducted in Spain, while 5 in France, 4 in Canada, 3 in America and 2 in Brazil. The median follow-up ranged from 3.5 to 19 years in cohort studies. The mean age of participants was between 28 and 69 years, exclusive of the unknown one [41]. The female proportion ranged from 49 to $100 \%$. Of the 20 eligible studies, 4 focused on allcause mortality [24-27], 2 on cardiocerebrovascular diseases [28, 29], 2 on metabolic syndrome [30, 31], 5 on overweight and obesity [16, 32-35], 2 on mental health diseases [36, 37]. The remaining 5 studies respectively investigated gastrointestinal diseases [38], cancers [39], pregnancy outcome [40], respiratory diseases [41], and geriatric diseases [42].

\section{Quality assessment}

The quality assessment was listed in Supplement 2. Cohort studies scored ranging from 6 to 9. A maximum of 9 points could be awarded to each cohort study: 4 for selection, 2 for comparability, and 3 for outcome. Two cohort studies represented the highest quality. The most common bias risk was incomplete representativeness. Volunteers [25, 28, 36, 38, 39] and university graduates $[29,35,37]$ tended to be more health-conscious and had

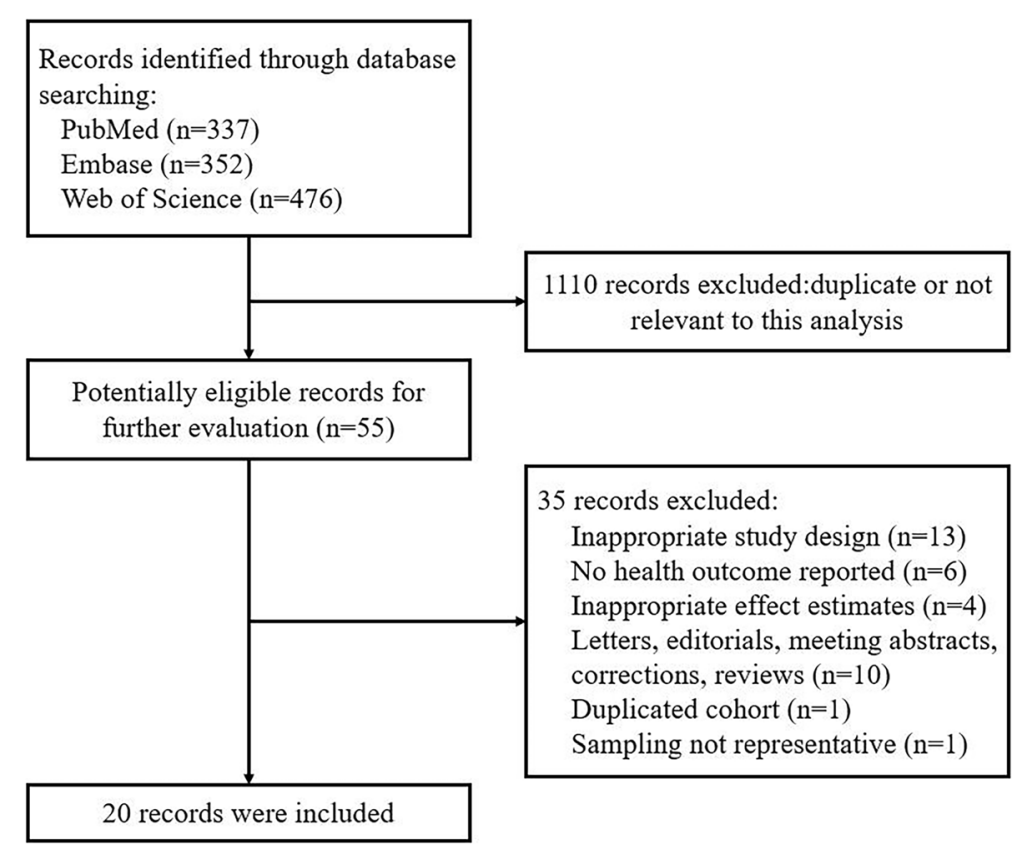

Fig. 1 Flowchart of literature search 


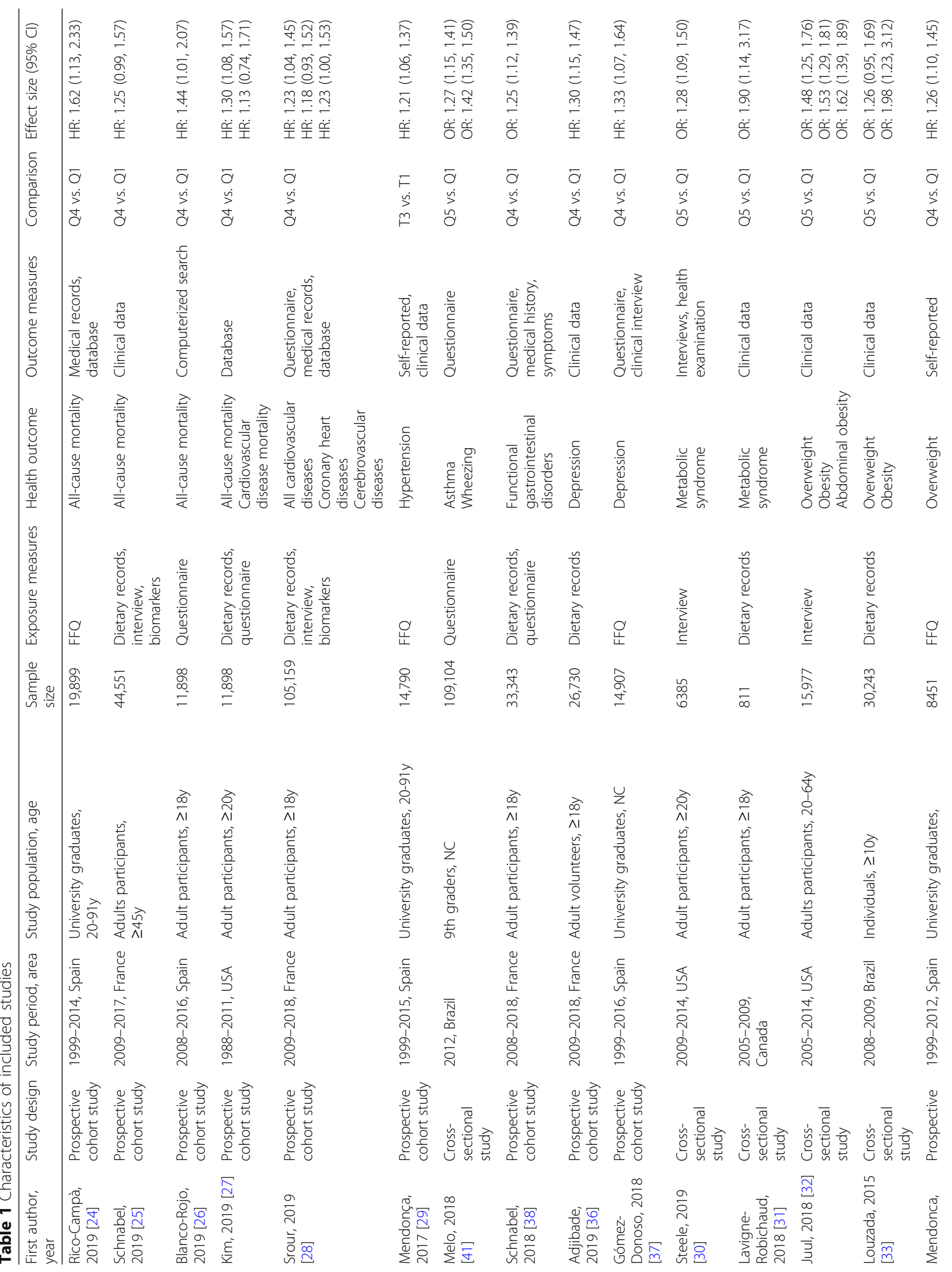




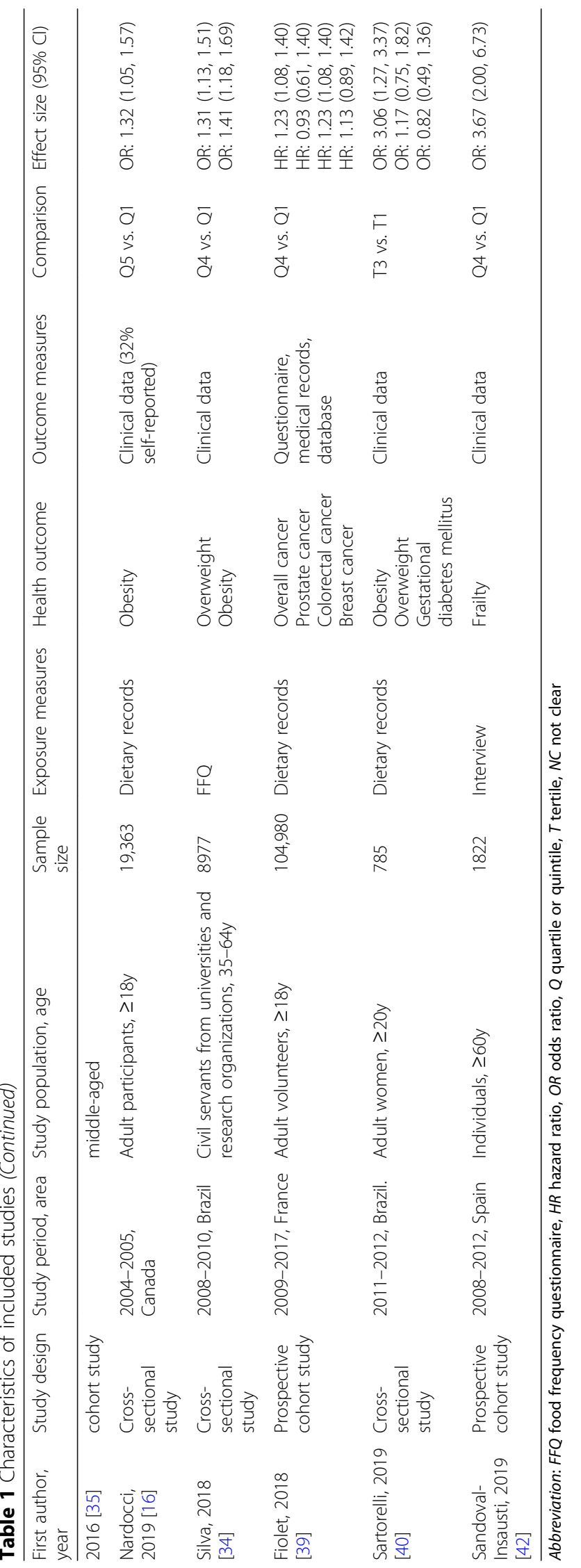


healthier dietary habits, leading to a lack of representativeness of the general population. Considering the chronic development of NCDs, the insufficient follow-up was another source of bias risk $[39,42]$.

Cross-sectional studies achieved 5 to 8 "yes". Three studies were unclear whether the measurement of the condition was assessed according to the objective and standard criteria [30, 31, 41]. Three studies applied incomplete statistical analysis [31, 34, 40]. The methods of exposures [33, 40] or outcomes [15, 41] measurement were short of validity and reliability, which led to bias risk. However, all the eligible studies made adequate adjustments for potential confounding factors. Generally, all included studies had a good methodological quality.

\section{Study results}

The results were synthesized in Table 1, comprising study design, study setting, samples, exposures, outcomes, and effect sizes. A narrative synthesis of our findings is as follows.

\section{All-cause mortality}

Four cohort studies investigated the association between UPFs consumption and risk of all-cause mortality [2427]. Despite diverse methods of exposures assessment, all studies reported a significant positive association, indicating that high consumption of UPFs was associated with an increased hazard for all-cause mortality. Three of them conducted sensitivity analyses and results did not substantially change, showing the strength of the association [24, 25, 27]. Rico-Campà et al. and Schnabel et al. found that cancer was the main cause of death. However, Kim et al. reported null association with cardiovascular disease mortality, which is surprising.

\section{Cardiocerebrovascular diseases}

Two cohort studies investigated the association between UPFs consumption and risk of cardiocerebrovascular diseases [28, 29]. Srour et al. focused on the overall of cardiovascular diseases, and Mendonca et al. on hypertension. Even after adjustment for potential confounding factors, it was found that high UPFs consumption increased the risk of overall cardiovascular diseases (HR: 1.23, $95 \%$ CI: 1.04 to 1.45 ), coronary heart diseases risk (HR: 1.18, 95\% CI: 0.93 to 1.52), cerebrovascular diseases risk (HR: $1.23,95 \%$ CI: 1.00 to 1.53), and hypertension (HR: $1.21,95 \%$ CI: 1.06 to 1.37 ). Results from sensitivity analyses did not substantially change.

\section{Respiratory diseases}

One cross-sectional study investigated the association between UPFs consumption and risk of asthma and wheezing among the Brazilian adolescents [41]. It found a positive association between UPFs consumption and risk of asthma (OR: $1.27,95 \% \mathrm{CI}: 1.15$ to 1.41 ) and wheezing (OR: $1.42,95 \%$ CI: 1.35 to 1.50 ). In addition, the direct association was stronger among male adolescents, those who did not consume fruits and vegetables regularly, non-smokers, with parents who did not smoke, and those living in non-capital cities.

\section{Gastrointestinal diseases}

One cohort study investigated the association between UPFs consumption and risk of functional gastrointestinal disorders [38]. In a sample of French adults, it was found that high UPFs consumption increased the risk of irritable bowel syndrome (IBS) (OR: 1.25, 95\% CI: 1.12 to 1.39) and concomitant functional dyspepsia (OR: 1.25 , 95\% CI: 1.05 to 1.47). No associations were observed between UPFs consumption and functional dyspepsia alone without concomitant IBS, indicating the indispensable role of IBS in the positive association.

\section{Mental health diseases}

Two cohort studies investigated the association between UPFs consumption and risk of depression [36, 37]. Both reported a positive association even after extensive adjustment. The fourth quartile had a significantly increased risk compared to the lowest quartile. Similar results were observed after sensitivity analyses, confirming the robustness of the association.

\section{Metabolic syndrome}

Two cross-sectional studies investigated the association between UPFs consumption and risk of metabolic syndrome [30, 31]. Both reported a significant positive association, suggesting the growing evidence of associations between UPFs consumption and several diet-related NCDs. In addition, Steele et al. observed that the association was stronger among young adults and decreased with age.

\section{Overweight and obesity}

Four cross-sectional studies [16, 32-34] and one prospective cohort study [35] investigated the association between UPFs consumption and risk of overweight $\left(\mathrm{BMI} \geq 25 \mathrm{~kg} / \mathrm{m}^{2}\right)$ and obesity $\left(\mathrm{BMI} \geq 30 \mathrm{~kg} / \mathrm{m}^{2}\right)$. Three studies reported a positive association for overweight $[32,33,35]$ and four studies for obesity [16, 32-34]. Furthermore, Juul et al. found a positive association between high UPFs consumption and abdominal obesity (OR: 1.62, 95\% CI: 1.39 to 1.89). Stronger effects were observed among women, partly due to sex-related differences in food choices [32,33]. The evidence strongly supported the role of increased UPFs consumption in the obesity epidemic worldwide. 


\section{Cancers}

One cohort study investigated the association between UPFs consumption and risk of cancers [39]. After a relatively short median follow-up of 5 years, this volunteerbased study suggested a positive association between UPFs consumption and overall cancer risk (HR: 1.23, 95\% CI: 1.08 to 1.40 ) and postmenopausal breast cancer risk (HR: $1.38,95 \%$ CI: 1.05 to 1.81 ). No significant association was observed for prostate, colorectal, overall breast and premenopausal breast cancers. However, a direct association about overall breast cancer risk was obtained when UPFs consumption was regarded as a continuous variable.

\section{Pregnancy outcome}

One cross-sectional study investigated the association between UPFs consumption and risk of several pregnancy outcomes [40]. This study was conducted among adult women with singleton pregnancies. It detected a positive association between UPFs consumption and pregnant obesity (OR: 3.06, 95\% CI: 1.27 to 3.37 ), but no significant association was observed in gestational diabetes mellitus (OR: 0.82, 95\% CI: 0.49 to 1.36) and overweight (OR: $1.17,95 \%$ CI: 0.75 to 1.82 ).

\section{Geriatric diseases}

One cohort study investigated the association between UPFs consumption and risk of incident frailty in the old adults [42]. With a relatively short median follow-up of 3.5 years, this study suggested a positive association between UPFs consumption and frailty risk (OR: 3.67, 95\% CI: 2.00 to 6.73$)$. Similar results were observed in sensitivity analyses.

\section{Discussion}

To the best of our knowledge, this is the first systematic review of available epidemiological evidence on the association between UPFs consumption and health outcomes. We identified 12 cohort and 8 cross-sectional studies, and found that there was a positive association between UPFs consumption and risk of all-cause mortality, overall cardiovascular diseases, coronary heart diseases, cerebrovascular diseases, hypertension, metabolic syndrome, overweight and obesity, depression, irritable bowel syndrome, overall cancer, postmenopausal breast cancer, gestational obesity, adolescent asthma and wheezing, and frailty. It showed no obvious association with cardiovascular disease mortality, prostate and colorectal cancer, gestational diabetes mellitus and gestational overweight.

Among the included studies, different methods were applied to estimate intake of UPFs. In some studies portion sizes were estimated using validated photographs. They calculated energy and weight relative to total food intake according to specific food composition databases. Some studies calculated daily intake by multiplying the portion size by the frequency of consumption, which had been proved validated. A majority of included studies evaluated intake as the percentage to total energy while the others selected weight proportion. The application of energy proportion contributed to reduction of variation due to body size, metabolic efficiency, and physical activity [33]. Weight proportion was taken into account for UPFs that did not provide any energy intake such as artificially sweetened drinks, and factors related to food processing [38]. Although methods vary, UPFs intake was all modeled as quantiles (e.g., tertiles, quartiles and quintiles). We selected effect estimates adjusted by the most factors for the highest versus the lowest consumption levels, which made them comparable. In light of the increasing concern of UPFs, further research is needed to set standard methods for UPFs intake estimation.

Despite different ways to estimate intake of UPFs, all the studies conducted food classification using the NOVA system, except one [33]. Louzada et al. divided foods into three groups according to the degree of processing, which was also consistent with the NOVA system. NOVA, a food classification system which classifies foods into four groups according to the nature, extent and purpose of industrial processing, has now been applied globally [49]. Groups are as follows: (i) Unprocessed or minimally processed foods; (ii) Processed culinary ingredients; (iii) Processed foods; (iv) Ultraprocessed foods. Instead of focusing on nutrient composition of the diet, it takes into consideration all physical, chemical and biological methods used during the food productive process [50]. The NOVA system has been used to describe population dietary patterns, assess changes in the dietary share of UPFs, and analyze the association of the dietary share with nutrient profile and with health outcomes [50]. It will contribute to the prevention of NCDs and the improvement of public health worldwide [51]. Nevertheless, when using the NOVA system to make dietary recommendations, it should be considered that some foods are difficult to classify [2]. Further research is needed to promote the application of this food classification system.

Considering the synergistic health-related effects of foods, it is of great importance to study dietary patterns instead of single foods or nutrients. UPFs consumption is increasing dominantly across the globe, especially in Western countries. It is consistent with the increased burden of NCDs attributable to unhealthy diets. The role of some specific UPFs has been assessed, such as processed meats and sweetened beverages, showing positive associations with NCDs $[52,53]$. In line with our findings, previous studies reported an inverse association 
between higher diet quality and risk of all-cause, cardiovascular disease, and cancer mortality [54-56]. Adherence to healthier diet patterns, which are characterized by a high consumption of unprocessed or minimally processed foods, was promoted to prevent NCDs. Decrease of cardiovascular disease burden with a healthier food system was observed in two modelling studies [57, 58]. A review of systematic reviews found that grain products and tea were protective, while processed meats and soft drinks tended to increase the risk [59]. Existing meta-analyses demonstrated that an optimal intake of several food groups could decrease the risk of coronary heart disease, stroke and heart failure [60]. Overall, UPFs consumption should be limited in prevention of NCDs.

The mechanism is multi-faceted. First, UPFs consumption is usually accompanied with high intake of fats, calories, sugars and salt, and low intake of micronutrients and fibre. The poor quality of dietary nutrients leads to the development of NCDs [61]. Processing, especially heat treatments, food additives and food packaging, can generate carcinogenicity and genotoxicity [25]. UPFs consumption increases added sugar intake, which is associated with obesity and several other health outcomes $[19,21,62,63]$. Moreover, higher intake of UPFs induces changes in gut microbiota, serum C-reactive protein levels and lipoprotein profiles [64-67]. Displacement of unprocessed or minimally processed foods might play a potential role in decreased diet quality [37]. However, it still remains unclear what plays a leading role in the association. A better understanding of what really matters and how various aspects contribute to the effects is highly needed.

Our study has strengths. To our knowledge, this is the most comprehensive systematic review of the topic to date. We carried out extensive literature research. The occurrence of selection bias was reduced greatly due to the prospective design of all the cohort studies. Large number of participants might compensate for the inadequate number of studies of each health outcome. All the eligible studies made adequate adjustments of the potential confounding factors. In general, we provide strong implications for dietary policies and guidelines.

Several limitations should also be acknowledged. First, most cohort studies recruited university graduates or volunteers as study objects, who tended to be more health-conscious and had a lower UPFs consumption than the general population. This probably resulted in an underestimation of the association of interest. Second, as occurrence of some health outcomes took a long time, such as carcinogenic processes, the median followup was relatively inadequate. Besides, complete detection of outcomes could not be guaranteed. Third, epidemiological studies could not exclude reverse causality and residual confoundings. For cross-sectional studies, probability existed that participants changed their dietary habits after the occurrence of diseases. It tended to cause an underestimation of the results. Fourth, some misclassification in the NOVA system could not be ruled out. Ways applied for dietary assessment were not specifically designed for NOVA classification and UPFs consumption yet. However, substantial differences between the highest and the lowest group might reduce the bias to a great extent. It is also worth mentioning that no quantitative meta-analysis was conducted, which we hoped to be overcome with further research. Given ethical issues of conducting randomized controlled trials of risk factors, more well-designed epidemiological studies are needed to confirm these findings.

There are significant public health implications in our study. To date, prevention and control of NCDs are becoming a growing concern. UPFs are beginning to be recognized as an emerging health risk. The positive association between UPFs consumption and adverse health outcomes provides insights into dietary policies and guidelines. Encouraging a decrease in UPFs consumption and an increase in the proportion of unprocessed or minimally processed foods are a direct way to resolve the issue. Food taxation and surveillance on food marketing still play a vital role. Dietary guidelines, in accordance with the shift of the global food system and health, are a necessity to slow the prevalence of NCDs.

\section{Conclusion}

This study indicated a positive association between UPFs consumption and risk of several health outcomes. Our results encouraged a decrease in UPFs consumption and an increase in the proportion of unprocessed or minimally processed foods, such as fruits and vegetables. Considering diet-related risk factors, we provided insights into NCDs occurrence and prevention. Large-scale prospective designed studies are needed to confirm our findings and to better understand the relative effects of various aspects in UPFs.

\section{Supplementary information}

Supplementary information accompanies this paper at https://doi.org/10. 1186/s12937-020-00604-1.

Additional file 1: Supplyment 1: Supplementary Text 1. Review protocol.

Additional file 2: Supplyment 2: Supplementary Table 1. Quality of cohort studies according to the Newcastle-Ottawa Scale (NOS). Supplementary Table 2. Quality of cross-sectional studies according to the JBI Critical Appraisal Checklist.

\section{Abbreviations}

UPFs: Ultra-processed foods; NCDs: Noncommunicable diseases; Cls: Confidence intervals; HRs: Hazard ratios; ORs: Odds ratios; RRs: Relative risks 


\section{Acknowledgments}

The authors thank the staff of the Department of Gastroenterology,

Zhongnan Hospital of Wuhan University, for their guidance and support.

\section{Authors' contributions}

$X C, Z Z, H Y$ conceived and designed the study. XC, ZZ, HY, PQ acquired data. $X C, Z Z, H Y, H W, F W$ analyzed data and interpreted results. XC, ZZ, HY wrote the paper. QZ, JF, JN reviewed and edited the manuscript. All authors read and approved the final manuscript.

\section{Funding}

National Key R\&D Program of China (2017YFC0112302).

\section{Availability of data and materials}

The datasets supporting the conclusions of this article are included within the article and its additional files.

\section{Ethics approval and consent to participate}

All included studies underwent institutional review board approval. All participants provided informed consent.

\section{Consent for publication}

Not applicable.

\section{Competing interests}

The authors declare that they have no competing interests.

\section{Author details}

'Department of Gastroenterology, Zhongnan Hospital of Wuhan University, No. 169, Donghu Road, Wuchang District, Wuhan 430071, Hubei Province, China. ${ }^{2}$ Hubei Clinical Center \& Key Lab of Intestinal \& Colorectal Diseases, Wuhan, China

\section{Received: 27 January 2020 Accepted: 12 August 2020}

Published online: 20 August 2020

\section{References}

1. Moubarac JC, Batal M, Louzada ML, Martinez SE, Monteiro CA. Consumption of ultra-processed foods predicts diet quality in Canada. Appetite. 2017;108: $512-20$

2. Ares $G$, Vidal L, Allegue G, Gimenez A, Bandeira E, et al. Consumers' conceptualization of ultra-processed foods. Appetite. 2016;105:611-7.

3. Costa LM, Martins AP, Canella DS, Baraldi LG, Levy RB, et al. Ultra-processed foods and the nutritional dietary profile in Brazil. Rev Saude Publica. 2015; 49:38.

4. Moubarac JC, Martins AP, Claro RM, Levy RB, Cannon G, et al. Consumption of ultra-processed foods and likely impact on human health. Evidence from Canada. Public Health Nutr. 2013:16(12):2240-8.

5. Louzada ML, Martins AP, Canella DS, Baraldi LG, Levy RB, et al. Impact of ultra-processed foods on micronutrient content in the Brazilian diet. Rev Saude Publica. 2015:49:45.

6. Scrinis G, Monteiro CA. Ultra-processed foods and the limits of product reformulation. Public Health Nutr. 2018;21(1):247-52.

7. Martinez SE, Monteiro CA. Association between dietary share of ultraprocessed foods and urinary concentrations of phytoestrogens in the US. Nutrients. 2017;9(3):209.

8. Vandevijvere $\mathrm{S}$, De Ridder K, Fiolet T, Bel S, Tafforeau J. Consumption of ultra-processed food products and diet quality among children, adolescents and adults in Belgium. Eur J Nutr. 2019:58(8):3267-78.

9. Martinez SE, Popkin BM, Swinburn B, Monteiro CA. The share of ultraprocessed foods and the overall nutritional quality of diets in the US: evidence from a nationally representative cross-sectional study. Popul Health Metrics. 2017:15(1):6.

10. Julia C, Martinez L, Alles B, Touvier M, Hercberg S, et al. Contribution of ultra-processed foods in the diet of adults from the French NutriNet-Sante study. Public Health Nutr. 2018;21(1):27-37.

11. Aguirre A, Borneo MT, El KS, Borneo R. Exploring the understanding of the term "ultra-processed foods" by young consumers. Food Res Int. 2019:115 535-40.

12. Ayton A, Ibrahim A. The dramatic rise of ultra-processed foods. BMJ. 2019; 366:14970
13. Moodie R, Stuckler D, Monteiro C, Sheron N, Neal B, et al. Profits and pandemics: prevention of harmful effects of tobacco, alcohol, and ultraprocessed food and drink industries. Lancet. 2013;381(9867):670-9.

14. Monteiro CA, Cannon G, Levy RB, Moubarac JC, Louzada ML, et al. Ultraprocessed foods: what they are and how to identify them. Public Health Nutr. 2019;22(5):936-41.

15. Bielemann RM, Santos LP, Costa C, Matijasevich A, Santos IS. Early feeding practices and consumption of ultraprocessed foods at 6 y of age: findings from the 2004 Pelotas (Brazil) birth cohort study. Nutrition. 2018;47:27-32.

16. Nardocci M, Leclerc B, Louzada M, Monteiro CA, Batal M, et al. Consumption of ultra-processed foods and obesity in Canada. Can J Public Health. 2019;110(1):4-14.

17. Baker P, Friel S. Food systems transformations, ultra-processed food markets and the nutrition transition in Asia. Glob Health. 2016:12(1):80.

18. Marron-Ponce JA, Tolentino-Mayo L, Hernandez-F M, Batis C. Trends in ultraprocessed food purchases from 1984 to 2016 in Mexican households. Nutrients. 2018;11(1):25.

19. Latasa P, Louzada M, Martinez SE, Monteiro CA. Added sugars and ultraprocessed foods in Spanish households (1990-2010). Eur J Clin Nutr. 2018; 72(10):1404-12.

20. Juul F, Hemmingsson E. Trends in consumption of ultra-processed foods and obesity in Sweden between 1960 and 2010. Public Health Nutr. 2015; 18(17):3096-107.

21. Cediel G, Reyes M, Da CLM, Martinez SE, Monteiro CA, et al. Ultra-processed foods and added sugars in the Chilean diet (2010). Public Health Nutr. 2018; 21(1):125-33.

22. Kelly B, Jacoby E. Public health nutrition special issue on ultra-processed foods. Public Health Nutr. 2018;21(1):1-04.

23. Forouzanfar MH, Afshin A, Alexander LT, Anderson HR, Bhutta ZA, et al. Global, regional, and national comparative risk assessment of 79 behavioural, environmental and occupational, and metabolic risks or clusters of risks, 1990-2015: a systematic analysis for the global burden of disease study 2015. Lancet. 2016;388(10053):1659-724.

24. Rico-Campa A, Martinez-Gonzalez MA, Alvarez-Alvarez I, de Deus MR, de la Fuente-Arrillaga C, et al. Association between consumption of ultraprocessed foods and all cause mortality: SUN prospective cohort study. BMJ 2019:365(11949). https://doi.org/10.1136/bmj.|1949.

25. Schnabel L, Kesse-Guyot E, Alles B, Touvier M, Srour B, et al. Association between Ultraprocessed food consumption and risk of mortality among middle-aged adults in France. JAMA Intern Med. 2019;179(4):490-8.

26. Blanco-Rojo R, Sandoval-Insausti H, López-Garcia E, Graciani A, Ordovás JM, et al. Consumption of ultra-processed foods and mortality: a National Prospective Cohort in Spain. Mayo Clin Proc. 2019:94(11):2178-88.

27. Kim H, Hu EA, Rebholz CM. Ultra-processed food intake and mortality in the USA: results from the third National Health and nutrition examination survey (NHANES III, 1988-1994). Public Health Nutr. 2019;22(10):1777-85.

28. Srour B, Fezeu LK, Kesse-Guyot E, Alles B, Mejean C, et al. Ultra-processed food intake and risk of cardiovascular disease: prospective cohort study (NutriNet-Sante). BMJ. 2019;365(11451). https://doi.org/10.1136/bmj.11451.

29. Mendonca RD, Lopes AC, Pimenta AM, Gea A, Martinez-Gonzalez MA, et al. Ultra-processed food consumption and the incidence of hypertension in a Mediterranean cohort: the Seguimiento Universidad de Navarra project. Am J Hypertens. 2017;30(4):358-66.

30. Steele EM, Juul F, Neri D, Rauber F, Monteiro CA. Dietary share of ultraprocessed foods and metabolic syndrome in the US adult population. Prev Med. 2019:125:40-8

31. Lavigne-Robichaud M, Moubarac JC, Lantagne-Lopez S, Johnson-Down L, Batal $M$, et al. Diet quality indices in relation to metabolic syndrome in an indigenous Cree (Eeyouch) population in northern Quebec. Canada Public Health Nutr. 2018;21(1):172-80

32. Juul F, Martinez-Steele E, Parekh N, Monteiro CA, Chang WW. Ultra-processed food consumption and excess weight among US adults. Brit J Nutr. 2018; 120(1):90-100

33. Da Costa Louzada ML, Baraldi LG, Steele EM, Bortoletto Martins AP, Canella DS, et al. Consumption of ultra-processed foods and obesity in Brazilian adolescents and adults. Prev Med. 2015;81:9-15.

34. Silva FM, Giatti L, de Figueiredo RC, Bisi Molina MDC, Cardoso LDO, et al. Consumption of ultra-processed food and obesity: cross sectional results from the Brazilian longitudinal study of adult health (ELSA-Brasil) cohort (2008-2010). Public Health Nutr. 2018;21(12):2271-9.

35. Mendonca RD, Pimenta AM, Gea A de la Fuente-Arrillaga C, MartinezGonzalez MA, et al. Ultraprocessed food consumption and risk of 
overweight and obesity: the University of Navarra Follow-up (SUN) cohort study. Am J Clin Nutr. 2016;104(5):1433-40.

36. Adjibade M, Julia C, Alles B, Touvier M, Lemogne C, et al. Prospective association between ultra-processed food consumption and incident depressive symptoms in the French NutriNet-Sante cohort. Bmc Med. 2019; 17(78). https://doi.org/10.1186/s12916-019-1312-y.

37. Gomez-Donoso C, Martinez-Gonzalez MA, Romanos-Nanclares A, RuizEstigarribia L, Mendonca R, et al. Ultra-processed food consumption and the incidence of depression in a mediterranean cohort: the seguimiento universidad de navarra project. Eur J Clin Invest. 2018;481(SI):169.

38. Schnabel L, Buscail C, Sabate JM, Bouchoucha M, Kesse-Guyot E, et al. Association between ultra-processed food consumption and functional gastrointestinal disorders: results from the French NutriNet-Sante cohort. Am J Gastroenterol. 2018;113(8):1217-28.

39. Fiolet T, Srour B, Sellem L, Kesse-Guyo E, Alles B, et al. Consumption of ultraprocessed foods and cancer risk: results from NutriNet-Sante prospective cohort. BMJ. 2018;360(k322). https://doi.org/10.1136/bmj.k322.

40. Sartorelli DS, Crivellenti LC, Candelas Zuccolotto DC, Franco LJ. Relationship between minimally and ultra-processed food intake during pregnancy with obesity and gestational diabetes mellitus. Cad Saude Publica. 2019;35(4): e49318.

41. Melo B, Rezende L, Machado P, Gouveia N, Levy R. Associations of ultraprocessed food and drink products with asthma and wheezing among Brazilian adolescents. Pediat Allerg Imm-Uk. 2018;29(5):504-11.

42. Sandoval-Insausti H, Blanco-Rojo R, Graciani A, López-García E, MorenoFranco B, et al. Ultra-processed Food Consumption and Incident Frailty: a prospective Cohort Study of Older Adults. J Gerontol. 2019;75(6):1126-33.

43. Marron-Ponce JA, Flores M, Cediel G, Monteiro CA, Batis C. Associations between consumption of ultra-processed foods and intake of nutrients related to chronic non-communicable diseases in Mexico. J Acad Nutr Diet. 2019;119(11):1852-65.

44. Rauber F, Da CLM, Steele EM, Millett C, Monteiro CA, et al. Ultra-processed food consumption and chronic non-communicable diseases-related dietary nutrient profile in the UK (2008(-)2014). Nutrients. 2018;10(5):587.

45. Machado PP, Steele EM, Levy RB, Sui Z, Rangan A, et al. Ultra-processed foods and recommended intake levels of nutrients linked to noncommunicable diseases in Australia: evidence from a nationally representative cross-sectional study. BMJ Open. 2019;9(8):e29544.

46. Stroup DF, Berlin JA, Morton SC, Olkin I, Williamson GD, et al. Meta-analysis of observational studies in epidemiology: a proposal for reporting. Metaanalysis of observational studies in epidemiology (MOOSE) group. JAMA. 2000;283(15):2008-12.

47. The Newcastle-Ottawa Scale (NOS) for assessing the quality of nonrandomised studies in meta-analyses.: Ottawa Hospital Research Institute; 2015. (Accessed July 28,2015, at www.ohri.ca/programs/dinical_ epidemiology/oxford.htm).

48. Checklist for Analytical Cross Sectional Studies.: The Joanna Briggs Institute; 2017. (Accessed 2017, at http://joannabriggs.org/research/critical-appraisaltools.html).

49. Lawrence MA, Baker PI. Ultra-processed food and adverse health outcomes. BMJ. 2019;365:12289.

50. Monteiro CA, Cannon G, Moubarac JC, Levy RB, Louzada M, et al. The UN decade of nutrition, the NOVA food classification and the trouble with ultraprocessing. Public Health Nutr. 2018;21(1):5-17.

51. Moubarac JC, Parra DC, Cannon G, Monteiro CA. Food classification systems based on food processing: significance and implications for policies and actions: a systematic literature review and assessment. Curr Obes Rep. 2014; 3(2):256-72

52. Imamura F, O'Connor L, Ye Z, Mursu J, Hayashino Y, et al. Consumption of sugar sweetened beverages, artificially sweetened beverages, and fruit juice and incidence of type 2 diabetes: systematic review, meta-analysis, and estimation of population attributable fraction. BMJ. 2015;351:h3576.

53. Vernooij R, Zeraatkar D, Han MA, El DR, Zworth M, et al. Patterns of red and processed meat consumption and risk for Cardiometabolic and Cancer outcomes: a systematic review and meta-analysis of cohort studies. Ann Intern Med. 2019;171(10):732-41.

54. Reedy J, Krebs-Smith SM, Miller PE, Liese AD, Kahle LL, et al. Higher diet quality is associated with decreased risk of all-cause, cardiovascular disease, and cancer mortality among older adults. J Nutr. 2014;144(6):881-9.

55. Alvarez-Alvarez I, Zazpe I, Perez DRJ, Bes-Rastrollo M, Ruiz-Canela M, et al. Mediterranean diet, physical activity and their combined effect on all-cause mortality: the Seguimiento Universidad de Navarra (SUN) cohort. Prev Med. 2018;106:45-52.

56. Schwingshackl L, Schwedhelm C, Galbete C, Hoffmann G. Adherence to Mediterranean Diet and Risk of Cancer: An Updated Systematic Review and Meta-Analysis. Nutrients. 2017;9(10):1063. https://doi.org/10.3390/nu9101063.

57. Moreira PV, Baraldi LG, Moubarac JC, Monteiro CA, Newton A, et al. Comparing different policy scenarios to reduce the consumption of ultraprocessed foods in UK: impact on cardiovascular disease mortality using a modelling approach. PLoS One. 2015;10(2):e118353.

58. Moreira PV, Hyseni L, Moubarac JC, Martins A, Baraldi LG, et al. Effects of reducing processed culinary ingredients and ultra-processed foods in the Brazilian diet: a cardiovascular modelling study. Public Health Nutr. 2018; 21(1):181-8.

59. Fardet A, Boirie Y. Associations between food and beverage groups and major diet-related chronic diseases: an exhaustive review of pooled/metaanalyses and systematic reviews. Nutr Rev. 2014;72(12):741-62.

60. Bechthold A, Boeing H, Schwedhelm C, Hoffmann G, Knuppel S, et al. Food groups and risk of coronary heart disease, stroke and heart failure: a systematic review and dose-response meta-analysis of prospective studies. Crit Rev Food Sci Nutr. 2019;59(7):1071-90.

61. Micha R, Penalvo JL, Cudhea F, Imamura F, Rehm CD, et al. Association between dietary factors and mortality from heart disease, stroke, and type 2 diabetes in the United States. JAMA. 2017;317(9):912-24.

62. Rauber F, Louzada M, Martinez SE, Rezende L, Millett C, et al. Ultraprocessed foods and excessive free sugar intake in the UK: a nationally representative cross-sectional study. BMJ Open. 2019;9(10):e27546.

63. Martinez SE, Baraldi LG, Louzada ML, Moubarac JC, Mozaffarian D, et al. Ultra-processed foods and added sugars in the US diet: evidence from a nationally representative cross-sectional study. BMJ Open. 2016;6(3):e9892.

64. Zobel EH, Hansen TW, Rossing P, von Scholten BJ. Global changes in food supply and the obesity epidemic. Curr Obes Rep. 2016;5(4):449-55.

65. Zinocker MK, Lindseth IA. The Western Diet-Microbiome-Host Interaction and Its Role in Metabolic Disease. Nutrients. 2018;10(3):365. https://doi.org/ 10.3390/nu10030365.

66. Lopes A, Araujo LF, Levy RB, Barreto SM, Giatti L. Association between consumption of ultra-processed foods and serum C-reactive protein levels: cross-sectional results from the ELSA-Brasil study. Sao Paulo Med J. 2019; 137(2):169-76

67. Rauber F, Campagnolo PD, Hoffman DJ, Vitolo MR. Consumption of ultraprocessed food products and its effects on children's lipid profiles: a longitudinal study. Nutr Metab Cardiovasc Dis. 2015;25(1):116-22.

\section{Publisher's Note}

Springer Nature remains neutral with regard to jurisdictional claims in published maps and institutional affiliations.

Ready to submit your research? Choose BMC and benefit from:

- fast, convenient online submission

- thorough peer review by experienced researchers in your field

- rapid publication on acceptance

- support for research data, including large and complex data types

- gold Open Access which fosters wider collaboration and increased citations

- maximum visibility for your research: over $100 \mathrm{M}$ website views per year

At BMC, research is always in progress.

Learn more biomedcentral.com/submissions 